\title{
Alimentación y enfermedades neurodegenerativas: una cuestión sociocientífica para desarrollar la habilidad argumentativa
}

\author{
María José Cortés Parra ${ }^{1}{ }^{(0)}$, Rodrigo Rodríguez Cepeda ${ }_{\text {Universidad Pedagogica Nacionat, Colombia }}^{\left({ }^{\circ}\right.}$
}

Autor de correspondencia: ${ }^{1}$ dqu_mjcortesp747@pedagogica.edu.co Recibido: 15 de agosto de 2020 Revisado: 25 septiembre de 2020 Aprobado: 19 de octubre de 2020 Publicado: 18 de mayo de 2021

\section{Resumen}

El artículo muestra los resultados de un trabajo de investigación cuyo objetivo fue favorecer el desarrollo de la habilidad argumentativa en un grupo de profesores en formación inicial del programa de Licenciatura en Química de la Universidad Pedagógica Nacional, mediante el diseño y la aplicación de una unidad didáctica sobre las implicaciones de la química de alimentos en el desarrollo y prevención de la enfermedad de Parkinson [EP] como una cuestión sociocientífica. Se implementaron actividades como preguntas abiertas, foros de discusión y búsqueda de posibles alternativas nutricionales en la prevención y tratamiento de la EP. En cada una de las etapas se identificó el nivel de argumentación y se analizó el contenido conceptual de cada argumento. Se destaca que los estudiantes lograron alcanzar un nivel de argumentación superior al inicial al aplicar los conocimientos adquiridos en la resolución y búsqueda de alternativas por medio de un aprendizaje activo y reflexivo. Construyeron argumentos basados en fundamentos teóricos y experimentales sobre diferentes aspectos de esta controversia.

Palabras clave: discurso, educación, alimentación, bioquímica, enfermedad del sistema nervioso 


\title{
Feed and neurodegenerative diseases: a socio-scientific issue for developing argumentative skills
}

\begin{abstract}
The article shows the results of a research project whose objective was to promote the development of argumentative skills in a group of trainee teachers of the Chemistry degree program at the National Pedagogical University, through the design and application of a didactic unit on the implications of food chemistry in the development and prevention of the Parkinson's disease [PD] as a socio-scientific issue. Activities such as open questions, discussion forums, and search for possible nutritional alternatives in the prevention and treatment for the PD were implemented. In each stage, the level of argumentation was identified, and the conceptual content of each argument was analyzed. It should be noted that the students were able to achieve a higher level of argumentation than the initial one by using the knowledge acquired in the solution and search for alternatives through active and reflective learning. They built arguments based on theoretical and experimental grounds on different aspects of this controversy.
\end{abstract}

Keywords: discourse, education, nutrition, biochemistry, nervous system disease

\section{Alimentação e doenças neurodegenerativas: uma questão sócio-científica para o desenvolvimento da capacidade argumentativa}

\section{Resumo}

O artigo apresenta os resultados da pesquisa cujo objetivo era promover o desenvolvimento de habilidades argumentativas num grupo de professores estagiários do programa da Licenciatura em Química da Universidade Pedagógica Nacional, através da concepção e aplicação de uma unidade didática sobre as implicações da química alimentar no desenvolvimento e prevenção da Doença de Parkinson [DP] como uma questão sóciocientífica. Atividades como perguntas abertas, fóruns de discussão e busca de possíveis alternativas nutricionais na prevenção e tratamento para a DP foram implementadas. Em cada etapa, identificou-se o nível de argumentação e analisou-se o conteúdo conceitual de cada argumento. Destaca-se que os estudantes foram capazes de alcançar um nível de argumentação superior ao inicial, aplicando os conhecimentos adquiridos na resolução e buscando alternativas através de um aprendizado ativo e reflexivo. Eles construíram argumentos baseados em fundamentos teóricos e experimentais sobre diferentes aspectos desta controvérsia.

Palavras-chave: discurso, educação, nutrição, bioquímica, doença do sistema nervoso 
Uno de los principales retos de la educación en ciencias es formar profesionales capaces de participar activamente en debates y discusiones científicas que busquen posibles soluciones o alternativas para situaciones controvertidas propias de su contexto. Esto posiciona a los estudiantes como contribuyentes activos de la sociedad, con habilidades que les permiten aportar científicamente en la toma de estas decisiones (Sadler, 2009). Sin embargo, de acuerdo con Martín-Gámez y Erduran (2018), en el aula de ciencias los estudiantes presentan dificultades al momento de construir y expresar argumentos que reflejen su posición para participar en actividades que involucren el discurso científico, lo cual se debe a que algunos profesores no incorporan prácticas que permitan el desarrollo de la habilidad argumentativa. De esta manera, es necesario que los profesores en formación inicial reflexionen sobre el papel de la argumentación en el aula de ciencias y sobre la importancia de incorporar estrategias didácticas que fortalezcan esta habilidad en los procesos de enseñanza.

En el contexto de educación en ciencias, la argumentación es una herramienta de importancia crítica en el crecimiento del conocimiento científico, ya que permite la articulación entre las explicaciones, los modelos, las teorías y la evidencia determinada, mediante el uso de órdenes y respaldos, dirigidos a la resolución de preguntas e involucrados en la adquisición del conocimiento (Jiménez-Aleixandre \& Erduran, 2007). En este sentido, las cuestiones sociocientíficas [CSC] fomentan espacios que favorecen el desarrollo de la habilidad argumentativa, mediante la construcción social y negociación de argumentos, en donde el diálogo y el debate permiten a los estudiantes cuestionar, justificar y evaluar el conocimiento, por medio del discurso científico (Martín-Gámez \& Erduran, 2018). Por lo tanto, la habilidad argumentativa se desarrolla mediante actividades de participación de los estudiantes, en las cuales se permita analizar y discutir el conocimiento científico.

En este sentido, se consideran como CSC los problemas sociales controvertidos con vínculos científicos y tecnológicos, donde se reconoce que la ciencia y la sociedad están directamente relacionadas (Sadler, 2009). El estudio de las enfermedades neurodegenerativas [END] puede abordarse como una CSC, debido a que enfrenta un escenario mucho más complejo que solo entender sus aspectos clínicos, ya que día a día impactan la vida de numerosas personas en el mundo, lo que implica una serie de factores vinculados a escasos avances científicos en la investigación de los mecanismos biológicos, bioquímicos y ambientales que inciden en su desarrollo, y a la poca información sobre la prevención y el tratamiento. Por otro lado, están los factores sociales, relacionados con el deterioro de la calidad de vida del paciente y de los cuidadores, la incapacidad laboral del enfermo y los elevados costos en tratamientos y en la atención sanitaria (Garcés, 2016), los cuales son desafíos sociales, económicos, científicos y educativos.

Dichos desafíos requieren de herramientas de enseñanza o estrategias didácticas que brinden los conocimientos necesarios para comprender estas END, no solo como una patología que afecta únicamente al paciente, sino como una problemática social que afecta gravemente diferentes sectores de la sociedad.

Desde esta perspectiva, la investigación busca que el alumno asimile y entienda conceptos y procesos bioquímicos implicados en las END, al involucrar al estudiante en estrategias metodológicas y experimentales que le permitan aplicar los conocimientos adquiridos 
en la resolución y búsqueda de alternativas para el tratamiento de END desde factores nutricionales. Esto supone la creación de un ambiente propicio para promover el desarrollo de la habilidad argumentativa mediante el estudio de la alimentación en el desarrollo y prevención de las END, por medio de un aprendizaje activo y reflexivo y de la construcción de argumentos basados en fundamentos teóricos y experimentales sobre diferentes aspectos de esta controversia.

\section{Metodología}

La presente investigación se desarrolló bajo un enfoque de metodología mixta, ya que integra el enfoque cualitativo y el cuantitativo. Está enmarcada en el contexto de investigación preexperimental sin grupo control. Se parte de que el enfoque cualitativo busca comprender la naturaleza del fenómeno a partir de las relaciones que convergen en ella. La investigación cuantitativa busca comprender el fenómeno y determinar la correlación entre las variables cuantificadas (Hernández et al., 2017).

\section{Población}

La investigación se aplicó en un grupo de veinticuatro estudiantes del ciclo de profundización del programa de Licenciatura en Química de la Universidad Pedagógica Nacional, en el espacio del Énfasis Disciplinar I: Química de Alimentos y Productos Naturales. Se tuvo en cuenta la relación del programa académico de este seminario con la estrategia implementada. Esta población estaba compuesta por 16 mujeres y 8 hombres, de entre 19 y 25 años.

\section{Fases de la investigación}

La metodología desarrollada se dividió en cinco fases:

Fase uno: recopilación de información. Una vez definidos el tema de trabajo y el planteamiento del problema, se procedió a realizar la consulta bibliográfica. Se usaron diferentes recursos como libros, revistas especializadas y bases de datos para buscar información sobre los ejes temáticos de la investigación.

Fase dos: diseño de la fase diagnóstica. Se diseñó y se aplicó la prueba de entrada (imagen 1) para orientar las actividades de la unidad didáctica. Con ella se pretendió identificar los conceptos previos de los estudiantes mediante la representación de su estructura conceptual, relacionada con los temas de química de alimentos y END, por medio de un mapa conceptual. La caracterización del nivel inicial de argumentación de los estudiantes se realizó mediante un texto escrito.

Fase tres: diseño de actividades. Una vez culminado el análisis de la fase diagnóstica, se realizó el diseño de las actividades de la unidad didáctica "La alimentación: alternativas en la prevención y tratamiento de la enfermedad de Parkinson [EP]: retos y oportunidades" (imagen 2). Se diseñaron actividades enfocadas en construir ambientes de argumentación. Se abordaron aspectos sociales, políticos, económicos, educativos, clínicos y nutricionales, con énfasis especialmente en estos últimos como alternativas para la posible prevención de END. 


\section{Imagen 1}

Instrumento de la fase diagnóstica

INSTRUMENTO N¹. PRUEBA DE ENTRADA.

Código

Semestre

Edad

Este instrumento esta construido por dos secciones, en ambas se pretende identificar sus conceptos previos relacionados con enfermedades neurodegenerativas y procesos biológicos:

La primera sección consta de la elaboración de un mapa conceptual y la segunda constituida por la elaboración de un texto argumentativo, donde adicionalmente se busca caracterizar su nivel inicial de la habilidad de argumentación.

Usted cuenta con un tiempo máximo de 25 minutos para la resolución de este instrumento. El cual debe resolver individualmente y sin la ayuda de fuentes de consulta externas.

1. Elaborar un mapa conceptual empleando los conceptos que se presentan a continuación. Si considera que se debe adicionar otros conceptos, está en libertad de hacerlo. Recuerde que los mapas conceptuales son una red de proposiciones entre conceptos organizados de forma jerárquica, que pueden estar unidos mediante frases breves o palabras de enlace que evidencian el significado de la relación conceptual.
a. Alimentos.
b. Compuestos bioactivos.
c. Aminoácidos
d. Proteínas
e. Enzimas
f. Inhibidores
g. Sitio activo

h. Catalizadores

i. Neuronas.

j. Neurotransmisores

k. Dopamina

I. Enfermedades neurodegenerativas

m. Enfermedad de Parkinson.

2. En un texto argumente el porqué de la estructura que le dio a este mapa conceptual, especificando como se relacionan biológicamente estos conceptos.

\section{Imagen 2}

Unidad didáctica

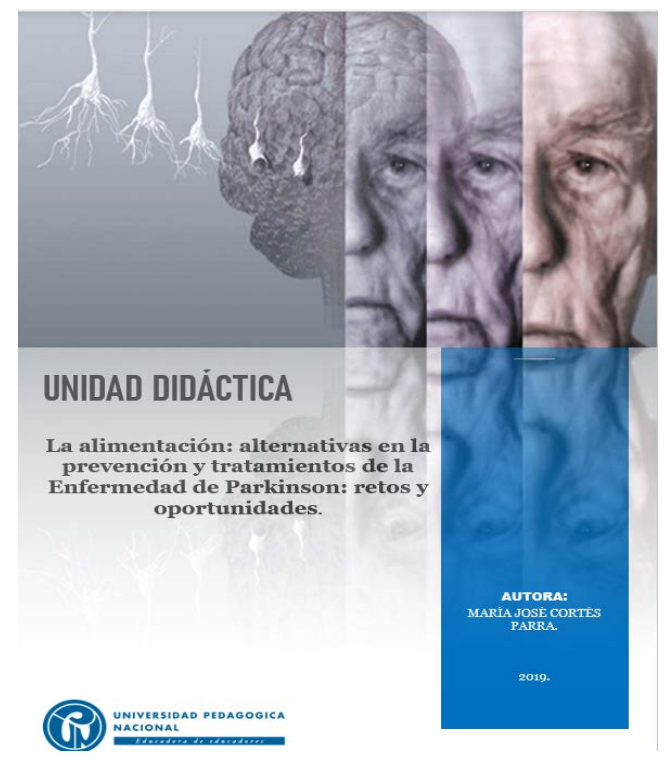


Fase cuatro: implementación de la unidad didáctica. La unidad didáctica se constituyó de seis actividades (imagen 3) de introducción, aprendizaje, desarrollo y aplicación, las cuales se implementaron en la población objeto de estudio.

\section{Imagen 3}

Contenidos de la unidad didáctica

\section{Objetivos de la Unidad Didáctica}

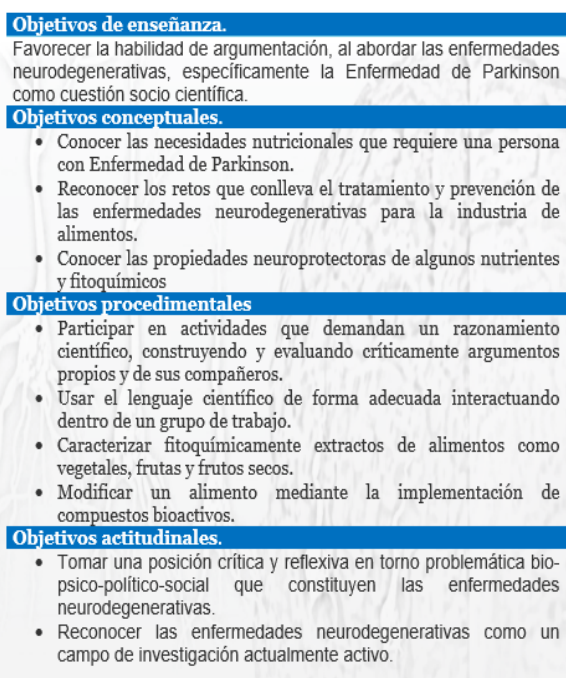

Reconocer los retos que conlleva el tratamiento y prevención de las enfermedades neurodegenerativas para la industria de alimentos.

- Conocer las propiedades neuroprotectoras de algunos nutrientes yitogúmicos

Objetivos procos

- Participar en actividades que demandan un razonamiento científico, construyendo y evaluando cíticamente angumentos propios y de sus compañeros.

propios de sus conpanies. dentro lenguje ciention

- dentro de un grupo de trabajo. pranos de a - Caracterizar fitoquímicamente

- vegetes, frutas y futos secos. Modificar un alimento mediante la implementación de compuestos bioactivos.

- Tomar una posición crítica y reflexiva en torno problemática bio- Tomar una posición critica y rellexiva en tomo problemática biopsico-político-social que constituyen las enfermedades neurodegenerativas.

Reconocer las enfermedades neurodegenerativas como un campo de investigación actualmente activo.

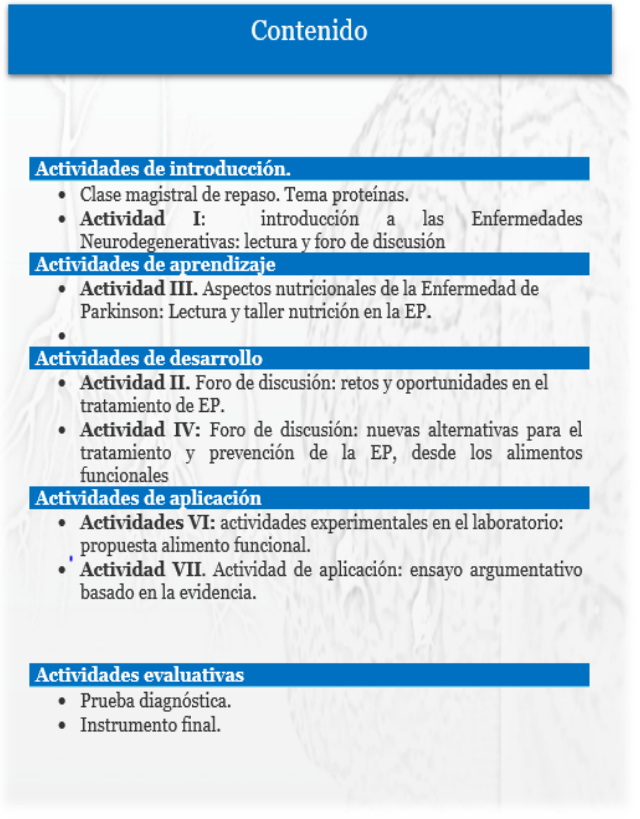

Fase cinco: evaluación de la eficacia de la unidad didáctica. Para finalizar, se evaluó la eficacia de la unidad didáctica. Se aplicó el mismo instrumento de entrada (imagen 1) y se compararon los resultados obtenidos por los estudiantes frente al nivel argumentativo inicial.

\section{Resultados}

\section{Fase diagnóstica}

Para el análisis de los resultados obtenidos en la parte inicial de la prueba diagnóstica, se tomaron como referencia los criterios para evaluar mapas conceptuales, establecidos por Costamagna (2001), los cuales se fundamentan en la teoría cognitiva del aprendizaje de Ausubel. Estos criterios fueron adaptados para la presente investigación. Cada ítem se evaluó y se valoró numéricamente de uno a cinco, como se evidencia en la tabla 1.

En cuanto al nivel de argumentación, tanto en la fase diagnóstica como en todas las etapas implementadas, se evaluó al tomar como referencia los niveles establecidos por Osborne, citados por Posada (2015) y modificados en esta investigación (tabla 2), los cuales se fundamentan en el modelo argumentativo propuesto por Toulmin. Por otra parte, se analizó el contenido conceptual de cada argumento, al realizar una triangulación de la información y recurrir a referentes teóricos para validar el contenido suministrado por los estudiantes. 
Tabla 1

Criterios para evaluar un mapa conceptual modificados por la autora

\begin{tabular}{|c|c|c|}
\hline Ítem & Aspecto para evaluar & $\begin{array}{l}\text { Valora- } \\
\text { ción }\end{array}$ \\
\hline \multirow{6}{*}{ 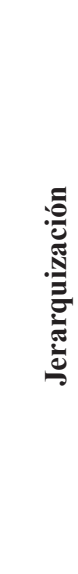 } & $\begin{array}{l}\text { Presenta una jerarquía estructurada, en la cual de forma clara y correcta incluye } \\
\text { todos los conceptos. No presenta errores al momento de definir y relacionar los } \\
\text { conceptos. }\end{array}$ & 5 \\
\hline & $\begin{array}{l}\text { La organización conceptual que presenta es simple, pero cada una de las definiciones } \\
\text { son claras y correctas. }\end{array}$ & 4 \\
\hline & No incluye todos los conceptos, pero presenta una jerarquización clara y sin errores. & 3 \\
\hline & $\begin{array}{l}\text { Presenta una organización clara, en la cual no incluye todos los conceptos y además } \\
\text { presenta errores. }\end{array}$ & 2 \\
\hline & $\begin{array}{l}\text { La organización jerárquica no es clara. No incluye todos los conceptos y presenta } \\
\text { errores. }\end{array}$ & 1 \\
\hline & $\begin{array}{l}\text { El mapa conceptual no presenta una estructura definida. Los conceptos no están } \\
\text { organizados de forma clara o jerárquica. }\end{array}$ & 0 \\
\hline \multirow{6}{*}{ 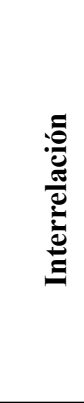 } & $\begin{array}{l}\text { Presenta de forma correcta varias relaciones cruzadas, mostrando uniones entre los } \\
\text { diferentes conceptos de un determinado tema. }\end{array}$ & 5 \\
\hline & $\begin{array}{l}\text { Incluye mínimo tres relaciones cruzadas entre diferentes partes del mapa de forma } \\
\text { clara y correcta. }\end{array}$ & 4 \\
\hline & Alguna de las relaciones cruzadas que presenta no es clara o correcta & 3 \\
\hline & Muestra dos o una relación cruzada de forma correcta. & 2 \\
\hline & Las relaciones cruzadas que presenta no son correctas. & 1 \\
\hline & No presenta ninguna relación cruzada. & 0 \\
\hline \multirow{6}{*}{ 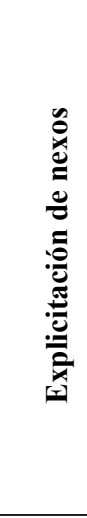 } & $\begin{array}{l}\text { Usa de forma apropiada oraciones nodales, frases y palabras de enlace, para mostrar } \\
\text { claramente las relaciones entre los conceptos. }\end{array}$ & 5 \\
\hline & $\begin{array}{l}\text { Emplea correctamente palabras-enlace o frases para indicar la relación entre los } \\
\text { conceptos. }\end{array}$ & 4 \\
\hline & $\begin{array}{l}\text { Algunas de las palabras-enlace o frases para indicar la relación entre los conceptos } \\
\text { no son claras o correctas. }\end{array}$ & 3 \\
\hline & $\begin{array}{l}\text { La mayoría de las palabras-enlace o frases para indicar la relación entre los } \\
\text { conceptos no son claras o correctas. }\end{array}$ & 2 \\
\hline & $\begin{array}{l}\text { Las palabras-enlace o frases para indicar la relación entre los conceptos son } \\
\text { incorrectas. }\end{array}$ & 1 \\
\hline & No usa palabras-enlace o frases para indicar la relación entre los conceptos. & 0 \\
\hline \multirow{6}{*}{ 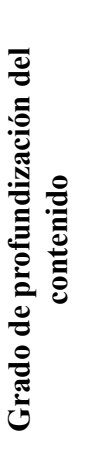 } & $\begin{array}{l}\text { Incluye detalles o ejemplos que permiten profundizar en mayor grado el contenido } \\
\text { del mapa conceptual. Adiciona algunos tipos de concepto que permita profundizar } \\
\text { más el mapa conceptual. }\end{array}$ & 5 \\
\hline & $\begin{array}{l}\text { Incluye al menos un ejemplo, detalle y varios conceptos de forma correcta para } \\
\text { profundizar en mayor grado el contenido del mapa conceptual. }\end{array}$ & 4 \\
\hline & $\begin{array}{l}\text { Incluye un ejemplo o detalle, o conceptos, de forma clara, para profundizar en mayor } \\
\text { grado el contenido del mapa conceptual. }\end{array}$ & 3 \\
\hline & Los conceptos, ejemplos o detalles que incluye en su mayoría son incorrectos. & 2 \\
\hline & Los conceptos, ejemplos o detalles que incluye en su totalidad son incorrectos. & 1 \\
\hline & No incluye detalles, ejemplos o conceptos adicionales. & 0 \\
\hline
\end{tabular}

Nota: adaptado de Costamagna (2001). 
Tabla 2

Niveles de argumentación modificados por la autora

\begin{tabular}{|c|c|}
\hline Nivel de argumentación & Características \\
\hline 0 & $\begin{array}{l}\text { Los argumentos presentan únicamente datos sin llegar a ninguna } \\
\text { conclusión. }\end{array}$ \\
\hline 1 & Los argumentos presentan únicamente datos y conclusiones. \\
\hline 2 & $\begin{array}{l}\text { Los argumentos tienen conclusiones, datos, calificativos y además } \\
\text { garantías. }\end{array}$ \\
\hline 3 & $\begin{array}{l}\text { Los argumentos tienen conclusiones, datos, garantías, calificativos y } \\
\text { respaldos basados en fuentes externas y resultados de investigación o } \\
\text { resultados experimentales. }\end{array}$ \\
\hline 4 & Los argumentos son completos y presentan una refutación clara. \\
\hline 5 & $\begin{array}{l}\text { Los argumentos son extensos y completos. Las garantías y el soporte } \\
\text { presentan refutaciones claras y estructuradas. }\end{array}$ \\
\hline
\end{tabular}

Nota: adaptado de Posada (2015).

De acuerdo con la estructura de estos mapas conceptuales, se identificaron las siguientes falencias conceptuales frente a los conceptos relacionados con química de alimentos y END. $\mathrm{Al}$ intentar relacionar las END y la EP con conceptos como alimentos, proteinas, enzimas, catalizadores, compuestos bioactivos, neuronas, entre otros, se obtuvo que:

- El 58 \% de los estudiantes relaciona la aparición de estas enfermedades o su posible tratamiento con el consumo de proteínas. Se evidencia que no poseen información suficiente sobre los aspectos nutricionales relacionados con las END.

- El 75 \% de estudiantes no incluyó el concepto neurotransmisor, lo cual puede indicar que su definición aún no es clara. Por lo tanto, tampoco lo es su relación bioquímica con los otros conceptos.

- El $63 \%$ de los estudiantes no relaciona de forma correcta los conceptos enzimas, sitio activo, catalizadores e inhibidores. Esto demuestra que no poseen conocimientos relacionados con actividad enzimática o que no son capaces de relacionar estos conceptos con la enfermedad.

Por otro lado, solo cinco estudiantes - $20 \%$ - incluyeron conceptos, ejemplos o detalles para profundizar la información suministrada de forma correcta. Algunos de estos detalles son: aporte calórico, aminoácidos esenciales y no esenciales, reacción química, células, pared celular y daño neurológico. La tabla 3 muestra un mapa conceptual realizado por un estudiante.

En cuanto a la caracterización de los argumentos (gráfica 1), catorce estudiantes no presentaron el texto argumentativo, por lo que se situaron en el nivel 0 de argumentación. De los diez estudiantes que elaboraron el texto, cuatro están en un nivel 1, puesto que acudieron a datos de la estructura del mapa conceptual en relación con procesos bioquímicos y además plantearon alguna conclusión. Tres estudiantes presentaron información no relacionada con procesos biológicos y justificaron de forma simple la organización del mapa conceptual. Por lo tanto, se situaron en un nivel 0. Solo un estudiante mostró datos, garantías y construyó una conclusión, lo que lo situó en un nivel 2 de argumentación. Por esto, en su mayoría $-70,83 \%$ - tienen un nivel inicial de argumentación correspondiente a 0 , ya que presentan 
datos, sin llegar a conclusiones estructuradas o no presentan texto argumentativo.

Tabla 3

Mapa conceptual. Instrumento de entrada

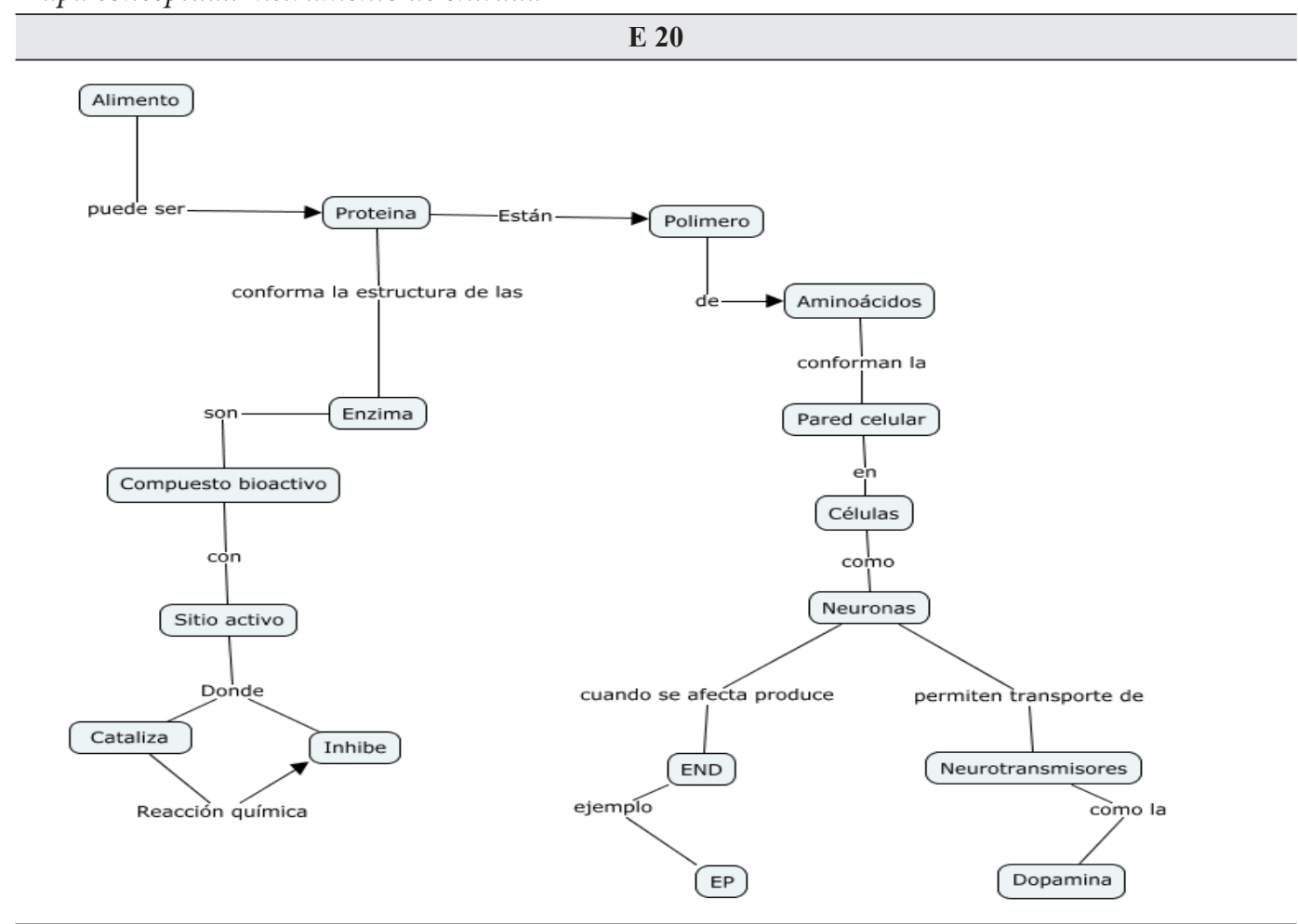

J. El mapa conceptual se divide en los conceptos generales, que son alimentos, proteínas y aminoácidos. Los errores que se presentan tienen que ver con la relación entre enzimas, compuesto bioactivo y sitio activo.

I. Se evidencia interrelación entre los conceptos sitio activo, cataliza e inhibe y reacciones químicas.

N. E. Utiliza frases y palabras conectoras para relacionar los conceptos de forma correcta. No emplea oraciones nodales. La única relación incorrecta es la de los conceptos compuesto bioactivo y enzima.

P. Emplea detalles como transporte de neurotransmisores. Especifica que el daño en las neuronas produce END. Adiciona conceptos como reacción química, pared celular y células.

Nota: estudiante [E], jerarquización [J], interrelación [I], explicitación de nexos [N. E.], grado de profundización del contenido [P].

\section{Gráfica 1}

Niveles de argumentación. Instrumento de entrada

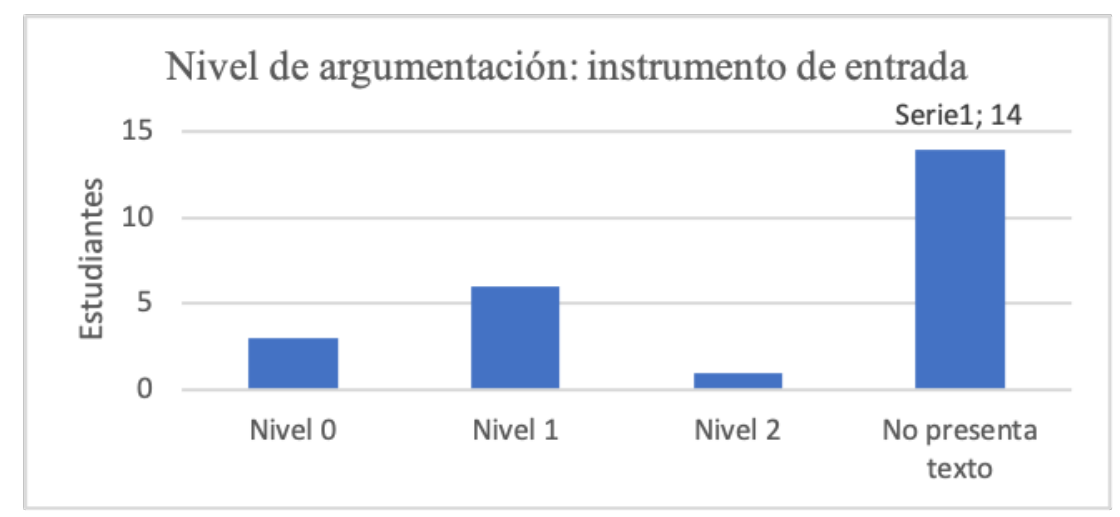


Por otra parte, para establecer si existe un nivel de correlación entre la estructura conceptual del estudiante y su nivel de argumentación, se calculó el coeficiente de correlación de Pearson, que mide el grado de asociación entre dos variables aleatorias cuantitativas, cuyos valores absolutos oscilan entre cero y uno, con el fin de determinar cómo se comporta un mismo grupo de personas en distintas variables (Reguant et al., 2018). Este cálculo se realizó en el paquete estadístico SPSS, para lo cual se emplearon como índices estadísticos la mediana de los ítems del mapa conceptual y el nivel de argumentación de cada estudiante. Así se obtuvieron los resultados que se muestran en la tabla 4.

Tabla 4

Correlación de Pearson

\begin{tabular}{cccc}
\hline & & Mapa Conceptual & Nivel de argumentación \\
\hline \multirow{2}{*}{ Mapa conceptual } & Correlación de Pearson & 1 &, $519^{* *}$ \\
& Sig. (bilateral) & &, 009 \\
& $\mathrm{~N}$ & 24 & 24 \\
\hline $\begin{array}{c}\text { Nivel de } \\
\text { argumentación }\end{array}$ & Correlación de Pearson &, $519^{* *}$ & 1 \\
& Sig. (bilateral) &, 009 & 24 \\
\hline
\end{tabular}

Nota: **la correlación es significativa al nivel 0,01 (bilateral).

El resultado indica que existe una correlación estadística significativa entre la estructura conceptual del estudiante, evidenciada en el mapa conceptual, y el nivel de argumentación, ya que el coeficiente es positivo y con un valor superior a 0,5 . De este resultado puede establecerse que a los estudiantes se les dificultó expresar su conocimiento sobre química de alimentos y END, tanto de forma textual como de forma representativa.

\section{Fase de implementación}

Actividad I. Teniendo en cuenta las falencias conceptuales identificadas en la fase diagnóstica, inicialmente se realizó una clase magistral de repaso, en la cual se abordó el tema de proteínas. En la segunda parte, se introdujo el tema de END mediante una lectura sobre los aspectos generales de estas patologías. A partir de esto, se realizó un foro de discusión en torno a la siguiente pregunta: “¿cuál considera que es el principal problema que se deriva de este tipo de enfermedades?".

Se resalta como resultado del foro que solo dos estudiantes se situaron en el nivel 1 de argumentación. Ocho estuvieron en el nivel 2 y trece alcanzaron un nivel 3, ya que presentaron argumentos mucho más elaborados y recurrieron a referentes científicos, estudios demográficos u opiniones de expertos. Sin embargo, ninguno empleó habilidades de alto nivel como las establecidas por Osborne et al. (2004), tales como producir órdenes de contrademanda o refutaciones. En la tabla 5, se presenta uno de los argumentos elaborados por los estudiantes correspondiente al nivel 3, así como su caracterización.

Respecto a los principales problemas derivados de las END, como se muestra en la gráfica 2, el $50 \%$ de los estudiantes identificó como principal problema social el impacto del deterioro en la calidad de vida del paciente, debido al deterioro físico y cognitivo que sufren las personas que padecen este tipo de patologías. De los resultados, se obtuvo que los estudiantes lograron contextualizarse con la situación actual de estas enfermedades. Reconocieron todas las implicaciones que trae consigo un aumento en su prevalencia, las 
cuales concuerdan con las mencionadas por Garcés (2016) en su estudio sobre el impacto de las END.

Tabla 5

Caracterización del argumento en actividad de introducción

\begin{tabular}{c} 
E 3 \\
Nivel de argumentación: 3 \\
\hline
\end{tabular}

de Michael Eduardo Prieto Cantuca - miércoles, 18 de septiembre de 2019, 20:36

Cuando hablamos de las enfermedades neurodegenerativas nos enfocamos en el daño celular que existe a nivel cerebral por distintas razones, así como menciona la señorita (Laura Vasquez en el caso del alzheimer que se produce por la presencia de la proteina beta amiloide), de la misma manera que puede ocurrir en otras enfermedades de la misma índole que afecta no solo las neuronas y las funciones del cuerpo humano, también afecta directamente el estilo de vida de una persona que abordan diversos problemas, como sociales, politicos, económicos, familiares etc... Sin embargo a mi punto de vista el mayor problema para una persona con alguna de estas enfermedades es el trato familiar pues desde alli es donde mas se enfoca la vida de una persona y es donde mas recae el apoyo. (Torrell Vallespin, 2019) Los miembros de la familia son, con frecuencia, los cuidadores primarios de las personas con trastornos mentales. Como tal, ellos proveen apoyo emocional $\mathrm{e}$ instrumental, y con no menor frecuencia también deben afrontar los costos financieros asociados con el tratamiento y la atención.

D. Menciona el deterioro neuronal causado por estas enfermedades, así como la forma en que se afecta la vida del paciente.

C. Indica que el mayor problema derivado de estas enfermedades está relacionado con la familia.

S. Como respaldo aborda las opiniones de una experta, sobre la enfermedad de Alzheimer.

G. Manifiesta que los cuidadores primarios generalmente son familiares y los costos que acarrean.

Q. "Los miembros de la familia son con frecuencia, los cuidadores"

Nota: estudiante [E], datos [D], garantías [G], conclusiones [C], calificadores [Q], respaldos [S], refutaciones [R].

\section{Gráfica 2}

Impactos de la prevalencia de las END identificados por los estudiantes.

\section{Principales problema derivado del aumento en la prevalencia de las END}

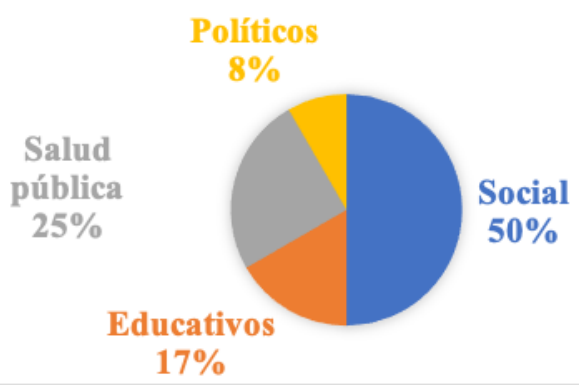

Actividad II. Conoce la EP: video explicativo. Para brindar los conceptos necesarios para la comprensión de la EP, se creó el video Enfermedad de Parkinson (Enfermedad ParkinsonCSC, 2019). Una vez observado el video, se les pidió a los estudiantes que, mediante un foro, respondieran la pregunta: “ ¿cuáles son los principales retos que abarca el tratamiento de la EP?" y comentaran por lo menos una de las respuestas de sus compañeros, 
para establecer un ambiente de discusión, y así conocer las diferentes posiciones y argumentos de los estudiantes y la forma como evalúan los argumentos de sus compañeros.

La caracterización de los argumentos permitió evidenciar que nueve estudiantes construyeron argumentos pertenecientes al nivel 3. Siete se ubicaron en el nivel 2 y cinco construyeron argumentos del nivel 1. La tabla 6 presenta uno de los textos escritos por los estudiantes.

De igual manera, se identificó que ocho estudiantes comentaron las respuestas de sus compañeros con el uso de operaciones de argumentación de un nivel superior. Tres de estos estudiantes refutaron las respuestas de sus compañeros y tres complementaron con información. Dos estudiantes contrademandaron los argumentos de otros con preguntas. Estos procesos se perciben como indicador significativo en la calidad de la argumentación, ya que, como plantea Osborne et al. (2004), se brinda la posibilidad de que los estudiantes evalúen su argumento y busquen evidencia que respalde su posición, para poder contrademandar o complementar.

Tabla 6

Argumentos del foro de discusión

E 17

Nivel de argumentación: 3

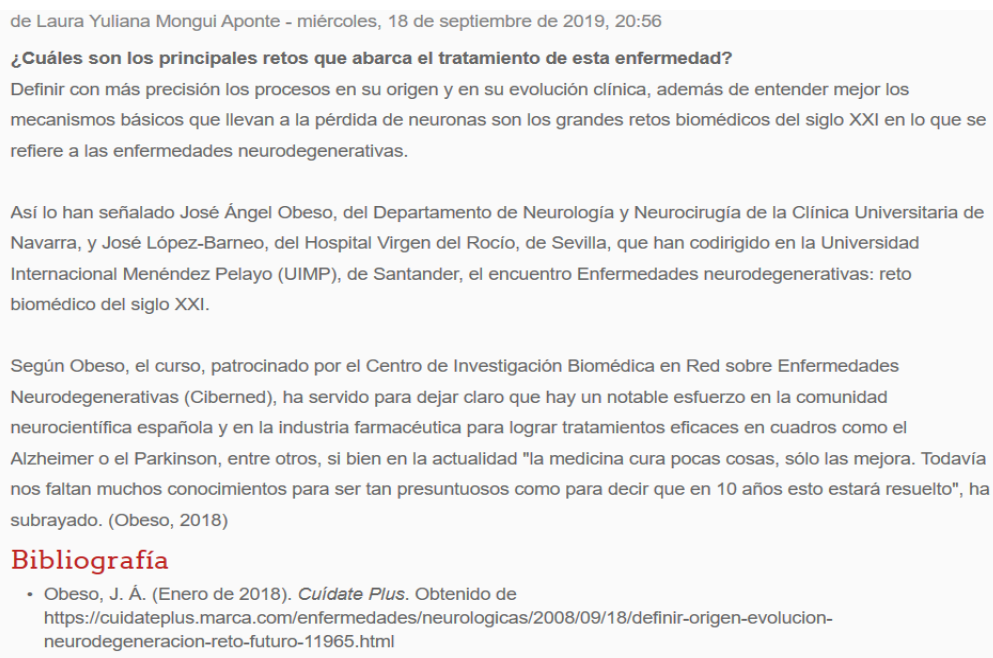

D. Contextualiza sobre la situación actual de la investigación sobre estas patologías.

G. Muestra los principales retos que definen algunas comunidades de neurólogos.

S. Recurre al juicio de expertos para abordar la situación actual de la investigación en las END en general.

C. Concluye que aún falta entender todos los procesos que causan las END, para llegar a una cura.

Nota: estudiante [E], datos [D], garantías [G], conclusiones [C], respaldos [S].

Como se evidencia en la gráfica 3, el 58 \% presentó como principal reto identificado en el tratamiento de la EP el relacionado con avances investigativos y estrategias educativas, vinculados con el entendimiento de estas patologías y los mecanismos bioquímicos que las causan. Estos retos coinciden con los establecidos por Fernández et al. (2016), los cuales indican que los tratamientos actuales solo controlan los síntomas y hasta el momento ninguno de los fármacos desarrollados ha demostrado efectos curativos. Por esto, se deben investigar nuevos fármacos con mejores características. 


\section{Gráfica 3}

Retos identificados en el tratamiento de la EP

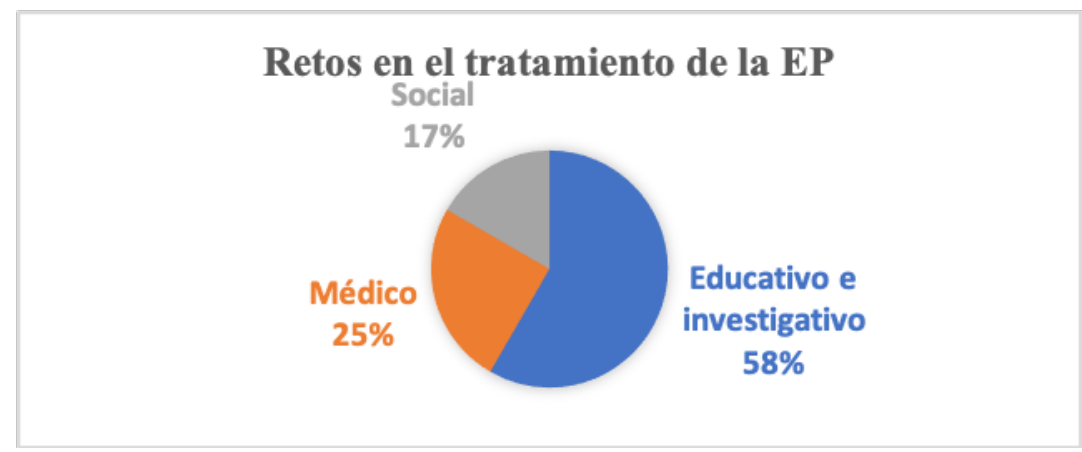

Actividad III. Aspectos nutricionales de la EP: taller sobre nutrición en la EP. El principal objetivo de esta actividad era que el estudiante reconociera la importancia de la alimentación en la prevención y especialmente en el tratamiento de la EP. A través de una lectura, se abordó la forma en que los nutrientes y micronutrientes intervienen en la evolución de esta enfermedad y en la acción del medicamento. Para ello, se les pidió a los estudiantes que realizaran una descripción de las características principales que deben cumplir los alimentos que pueden consumir las personas con EP, teniendo en cuenta los requerimientos nutricionales asociados a los síntomas primarios y secundarios. De esto se obtuvo que las características planteadas se pueden dividir en cuatro factores, presentados en la tabla 7.

Tabla 7

Resultados del taller de aspectos nutricionales en la EP

\begin{tabular}{|c|c|c|c|}
\hline $\begin{array}{l}\text { Característica } \\
\text { del alimento }\end{array}$ & Parámetro & $\begin{array}{c}\text { Cantidad de } \\
\text { estudiantes que } \\
\text { lo mencionaron }\end{array}$ & $\begin{array}{c}\text { Síntoma o requerimiento de la } \mathrm{EP} \text { con que se } \\
\text { relaciona }\end{array}$ \\
\hline \multirow{3}{*}{ Consistencia } & Textura & 8 & \multirow{3}{*}{$\begin{array}{l}\text { Ya que uno de los síntomas secundarios que } \\
\text { presenta esta patología es la disfagia - que es la } \\
\text { dificultad para mover los alimentos en la boca-, } \\
\text { se debe considerar que los alimentos ingeridos } \\
\text { deben ser blandos y de texturas húmedas }\end{array}$} \\
\hline & Dureza & 5 & \\
\hline & $\begin{array}{l}\text { Contenido de } \\
\text { humedad }\end{array}$ & 5 & \\
\hline \multirow{3}{*}{ Presentación } & Sabor & 3 & \multirow{3}{*}{$\begin{array}{l}\text { Este factor es relacionado con la motivación ha- } \\
\text { cia el paciente para consumir alimentos, ya que } \\
\text { uno de los síntomas secundarios es depresión } \\
\text { y falta de motivación, en la cual los pacientes } \\
\text { presentan poco interés en comer. }\end{array}$} \\
\hline & Olor & 2 & \\
\hline & Color & 2 & \\
\hline \multirow{5}{*}{$\begin{array}{c}\text { Valor } \\
\text { nutricional }\end{array}$} & $\begin{array}{l}\text { Contenido de } \\
\text { proteína }\end{array}$ & 18 & \multirow{5}{*}{$\begin{array}{l}\text { Considerando los requerimientos como aporte } \\
\text { calórico, se debe tener especial cuidado con el } \\
\text { consumo de carbohidratos, con la cantidad de } \\
\text { proteínas para mantener la masa muscular, con } \\
\text { el consumo de fibra para evitar estreñimiento y } \\
\text { con los micronutrientes para fortalecer la masa } \\
\text { ósea }\end{array}$} \\
\hline & $\begin{array}{l}\text { Contenido de } \\
\text { fibra }\end{array}$ & 17 & \\
\hline & $\begin{array}{l}\text { Contenido de } \\
\text { carbohidrato }\end{array}$ & 9 & \\
\hline & $\begin{array}{l}\text { Contenido de } \\
\text { ácidos grasos }\end{array}$ & 9 & \\
\hline & $\begin{array}{l}\text { Contenido de } \\
\text { micronutrientes }\end{array}$ & 3 & \\
\hline
\end{tabular}




\begin{tabular}{|c|c|c|c|}
\hline \multirow{4}{*}{$\begin{array}{l}\text { Consumo de } \\
\text { alimentos con } \\
\text { vitaminas o } \\
\text { antioxidantes }\end{array}$} & Vitaminas & 12 & \multirow{4}{*}{$\begin{array}{l}\text { Se relacionó con las propiedades neuroprotec- } \\
\text { toras de los compuestos bioactivos, capaces de } \\
\text { mitigar la acción causada por radicales libres. } \\
\text { Por esto, algunos estudiantes consideraron que } \\
\text { los alimentos, además de cumplir con los ante- } \\
\text { riores requerimientos nutricionales, debían tener } \\
\text { estos compuestos adicionales. }\end{array}$} \\
\hline & Flavonoides & 7 & \\
\hline & Polifenoles & 2 & \\
\hline & Antioxidantes & 9 & \\
\hline
\end{tabular}

A partir de los resultados, fue posible evidenciar que los estudiantes relacionaron los requerimientos nutricionales con los efectos bioquímicos y sintomáticos secundarios de la EP. También identificaron las necesidades de los pacientes de forma correcta, ya que concuerdan con los establecidos por Mesa (2010). Entre estos, destaca la necesidad de tener en cuenta las posibles alteraciones que puede tener el consumo de altas cantidades de proteína con la cinética del medicamento levodopa, empleado en el tratamiento de la enfermedad, y el contenido de fibra para reducir algunos síntomas secundarios relacionados con estreñimiento. Así mismo, destaca la importancia del consumo de micronutrientes como vitaminas y minerales como posibles factores de prevención.

Actividad IV. Foro de discusión: nuevas alternativas para el tratamiento y prevención de la EP, desde los alimentos funcionales. En esta actividad, mediante un video explicativo (HablemosClaroTV, 2017) se abordó la temática de alimentos funcionales, compuestos bioactivos y neuroprotección, sobre el cual se desarrolló un debate alrededor de las preguntas: “¿aprobarías la venta y distribución en las tiendas de barrio de alimentos funcionales que contribuyan a la prevención y el tratamiento de las enfermedades neurodegenerativas? ¿Por qué?". Esto tuvo el objetivo de que los estudiantes defendieran sus ideas o refutaran las de sus compañeros, al acudir a la información presentada y su posición crítica frente al consumo y fabricación de alimentos funcionales para la prevención y tratamiento de las END, especialmente de la EP.

En esta actividad, tres estudiantes justificaron su respuesta mediante argumentos elaborados. Acudieron a evidencias y refutaciones, lo que los posicionó en el nivel 4 (tabla 8). Doce se situaron en un nivel 2; dos estudiantes, en el nivel 3; y tres, en el nivel 1. Por otro lado, al momento de evaluar los argumentos de sus compañeros, solo el estudiante 19 produjo órdenes de contrademanda y se ubicó en un nivel 5 de argumentación. Catorce estudiantes, al comentar los argumentos de sus compañeros, complementaban lo expuesto mediante habilidades argumentativas del nivel 2 .

Delos estudiantes, $52,38 \%$, estuvo de acuerdo en la distribución de alimentos modificados como alternativas en la prevención de la EP. Es importante recalcar que, cuando los estudiantes indicaban estar de acuerdo, algunos — seis- indicaban que su distribución se debería realizar con un respaldo de investigaciones que realmente demuestren sus beneficios. Otros - seis - lo aprobarían como alternativa de prevención de estas enfermedades, ya que se daría la posibilidad de estar al alcance de cualquier persona. El 38,09 \% manifestó no estar de acuerdo con la distribución de estos alimentos. Indicaron que ciertos alimentos naturalmente pueden aportar los mismos beneficios que los alimentos modificados y son más económicos y sin aditivos.

Actividad V. Aplicación en el aula del laboratorio de caracterización fitoquímica de extractos naturales. Con el fin de propiciar ambientes de construcción colectiva y 
búsqueda de soluciones, se pidió que, de acuerdo con los requerimientos nutricionales de la EP, buscaran un alimento que pudieran modificar mediante la adición de compuestos bioactivos que contribuyeran a la neuroprotección, de tal forma que sirviera como posible vía de prevención y tratamiento natural para esta patología.

Para ello, los estudiantes se organizaron en siete grupos de laboratorio y se les brindó información sobre los compuestos fitoquímicos, principales fuentes de extracción y propiedades neuroprotectoras, para que pudieran seleccionar una buena fuente de estos compuestos. Los extractos naturales y los alimentos propuestos por cada grupo de laboratorio se presentan en la tabla 9.

\section{Tabla 8}

Argumentos del foro de alimentos

\begin{tabular}{l} 
E 8 \\
Nivel de argumentación:4 \\
\hline 3. ¿Aprobarias la venta y distribución en las tiendas de barrio, de alimentos funcionales que contribuyan a la \\
prevención y el tratamiento de las enfermedades neurodegenerativas? ¿Por qué? Lo aprobaria como un proyecto de \\
prevención frente a las enfermedades neurodegenerativas, teniendo en cuenta también los muchos beneficios que \\
aportan al consumidor, sin embargo, considero que no se debería exceder su consumo, puesto que, si bien \\
mencionan en el video, si te tiene una dieta sana y balanceada, no hace falta el consumo de estos alimentos \\
funcionales; considero que las personas que tienen alguna enfermedad neurodegenerativa o algún déficit que estos \\
alimentos puedan mejorar, deben hacer consumo de estos de una manera consciente y medida. Por último, es \\
importante recalcar que, para la venta y distribución, se resalta el precio con el cual se comercializarán dichos \\
alimentos, pues si son para consumo de toda la población deben tener un precio asequible. \\
Referencias \\
Fuentes, L., Acevedo, D., \& Gelvez, V. M. (Julio-Diciembre de 2015). ALIMENTOS FUNCIONALES: IMPACTO Y \\
RETOS PARA EL DESARROLLO Y BIENESTAR DE LA sOCIEDAD COLOMBIANA. Biotecnología en el sector \\
Agropecuario y Agroindustrial, 13(2), 140-149. Obtenido de http://www.scielo.org.co/pdf/bsaa/v13n2/v13n2a16.pdf \\
\hline D. Aborda los beneficios que aportan al consumidor. \\
\hline C. Concluye que lo abordaría como un proyecto de prevención accesible para cualquier persona. \\
\hline G. Se deben consumir de manera consiente. \\
\hline Q. "Deben hacer consumo de estos de manera consiente y medida". \\
\hline R. Por otro lado, indica que no hace falta el consumo de estos alimentos si se tiene una dieta balanceada. \\
\hline S. Acude a la información suministrada en el video para plantear la refutación. \\
\hline Nota: estudiante [E], datos [D], garantías [G], conclusiones [C], calificadores [Q], respaldos [S], refu- \\
taciones [R].
\end{tabular}

\section{Tabla 9}

Alimentos y extractos de cada grupo de laboratorio

\begin{tabular}{ccc}
$\begin{array}{c}\text { Grupo de } \\
\text { laboratorio }\end{array}$ & Extracto natural de compuestos bioactivos & Propuesta de alimento modificado \\
\hline $\mathbf{1}$ & Cáscara de mandarina & Gelatina en polvo \\
\hline $\mathbf{2}$ & Almendras & Avena \\
\hline $\mathbf{3}$ & Almendras & Pan \\
\hline $\mathbf{4}$ & Cáscara de tomate de árbol & Batido verde \\
\hline $\mathbf{5}$ & Cáscara de pulpa de uva & Pechuga de pollo \\
\hline $\mathbf{6}$ & Remolacha & Gelatina sin sabor \\
\hline $\mathbf{7}$ & Quinua & Yogur \\
\hline
\end{tabular}

Estas actividades experimentales se dividieron en dos prácticas de laboratorio. En la 
primera, los estudiantes realizaron la extracción de compuestos fitoquímicos y, en la segunda práctica de laboratorio, hicieron una marcha fitoquímica, con la que lograron caracterizar compuestos bioactivos presentes en cada extracto, como saponinas, alcaloides, flavonoides y antraquinonas, mediante pruebas cualitativas. Esto les permitió a los estudiantes no solo identificarlos, sino también reconocer las propiedades neuroprotectoras de cada uno de los compuestos al momento de realizar el análisis de resultados.

Actividad VI. Elaboración de ensayo argumentativo basado en la evidencia. Teniendo en cuenta los resultados obtenidos experimentalmente, así como la información suministrada durante el desarrollo de la unidad didáctica, se pidió elaborar un ensayo en el cual abordaran las propiedades de cada alimento y las razones por las cuales este alimento puede contribuir a la prevención o tratamiento de END, como la EP. En esta actividad se pretendía que los estudiantes construyeran argumentos más claros, estructurados y respaldados, con base en los conocimientos adquiridos, el contexto social y la evidencia científica, obtenida experimentalmente y analizada en investigaciones científicas.

Los resultados mostraron que los estudiantes presentan argumentos cada vez más extensos, estructurados y claros. Se identificó que quince estudiantes estaban en nivel 3 de argumentación; cuatro, en un nivel 4; y solo un estudiante presentó un texto estructurado, con más de una refutación, lo que corresponde a un nivel 5. Por otro lado, un estudiante estuvo en el nivel 2. Ninguno realizó escritos correspondientes a un nivel 1 de argumentación.

De estos estudiantes, el 72 \% relacionó de forma adecuada la nutrición con las END. Mencionaron importantes características de los compuestos bioactivos que pueden incidir en la prevención de estas patologías. El 68 \% presentó información correcta sobre las propiedades neuroprotectoras de los fitoquímicos identificados experimentalmente. El 91 \% se basó en los resultados experimentales para justificar cómo su alimento modificado puede emplearse en el tratamiento o prevención de estas enfermedades. En la tabla 10, se presentan algunos de los componentes caracterizados en algunos de los ensayos presentados por los estudiantes.

Tabla 10

Estructura del argumento presentado en el ensayo

E 1

Nivel de argumentación: 4

D. Presenta datos claros y correctos sobre la síntesis de la dopamina, su función en el organismo y la importancia de esta.

C. Señala la gravedad de que los niveles de dopamina bajen en el organismo, así como la importancia del consumo de remolacha.

Q. La remolacha tiene niveles elevados de betaína.

G. Aborda los valores nutricionales de la remolacha, como contenido de proteína, hidratos de carbono y calorías, su contenido de vitaminas y aminoácidos esenciales. Resalta el contenido de betaína en la remolacha, compuesto altamente estudiado por sus propiedades neuroprotectoras en enfermedades como el alzhéimer.

S. Como soporte a lo anterior, acude a los resultados obtenidos experimentalmente en donde indica que se determinó la presencia de manera cualitativa de saponinas, alcaloides, antraquinonas, taninos, flavonoides y catequinas, en el extracto de remolacha. Esto lo complementa al acudir a referentes bibliográficos, en donde establece que la remolacha tiene propiedades neuroprotectoras.

R. Señala que un consumo elevado de remolacha puede contribuir a la formación de cálculos renales o contribuir a la acumulación de hierro

Nota: estudiante [E], datos [D], garantías [G], conclusiones [C], calificadores [Q], respaldos [S], refutaciones $[R]$. 


\section{Fase evaluación de la eficacia de la unidad didáctica}

Con el fin de determinar la eficacia y las implicaciones de la unidad didáctica, se implementó de nuevo el instrumento de entrada (imagen 1). Referente a los niveles de argumentación identificados en estos textos argumentativos, como se muestra en la gráfica 4, el mayor nivel de argumentación alcanzado fue 3, ya que solo siete estudiantes apoyaron sus justificaciones mediante referentes bibliográficos, como datos suministrados por investigaciones científicas o referentes teóricos, para definir algunos conceptos. Catorce estudiantes estuvieron en el nivel 2. Ellos recurrieron a datos para construir conclusiones. Para la construcción de argumentos, los estudiantes se basaron en conocimientos científicos relacionados con la función bioquímica de cada concepto.

\section{Gráfica 4}

Nivel de argumentación. Instrumento final

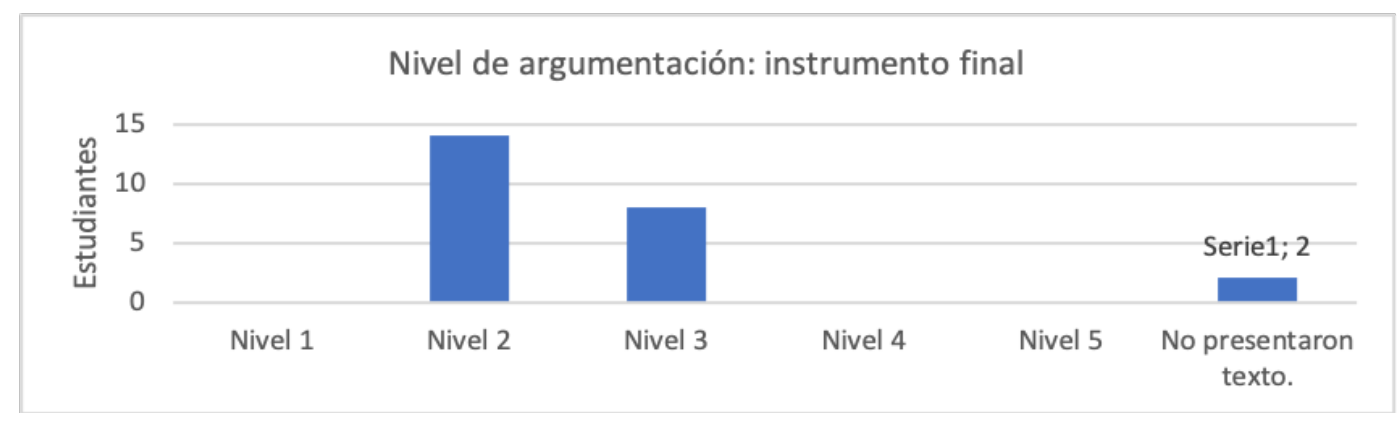

Frente a los mapas conceptuales construidos por los estudiantes, se puede evidenciar que en gran medida fueron superadas las falencias conceptuales identificadas al inicio de esta investigación, debido a que el 82,60 \% relacionó de forma correcta los conceptos EP y END con los conceptos relacionados con la química de alimentos, así como con los conceptos neuronas, neurotransmisor, dopamina, inhibidores y catalizadores. Un $74 \%$ incluye acertadamente el concepto proteina. De igual forma, la mayoría de los estudiantes $-83,33 \%$ - aborda correctamente el concepto compuesto bioactivo. Sin embargo, dos estudiantes siguen indicando que los aminoácidos o proteínas poseen sitio activo. Esto puede atribuirse a que no se profundizó en el concepto de estructura de enzimas.

En cuanto a los detalles que los estudiantes incluyen para complementar el mapa conceptual, se encuentra que $29,16 \%$ de los estudiantes incluyó conceptos relacionados con el mecanismo de acción del tratamiento farmacológico y sus principios bioquímicos como: levodopa, enzima DOPA descarboxilasa, carbidopa y benserazida. El 58,33 \% incluyó conceptos relacionados con la síntesis y la importancia de la dopamina. Involucraron conceptos como: L-tirosina, cerebro, neuronas dopaminérgicas y la importancia de este neurotransmisor. Uno de los mapas conceptuales realizados por los estudiantes se presenta en la tabla 11.

$\mathrm{Al}$ igual que en el instrumento de entrada, se determinó el nivel de correlación entre la estructura conceptual del estudiante y su nivel de argumentación en esta etapa de la investigación. Igualmente, el cálculo se realizó en el paquete estadístico SPSS y se usaron como datos la mediana de los ítems del mapa conceptual y el nivel de argumentación de 
cada estudiante. Los resultados obtenidos se presentan en la tabla 12.

\section{Tabla 11}

Mapas conceptuales instrumento de entrada.

\section{E 5}

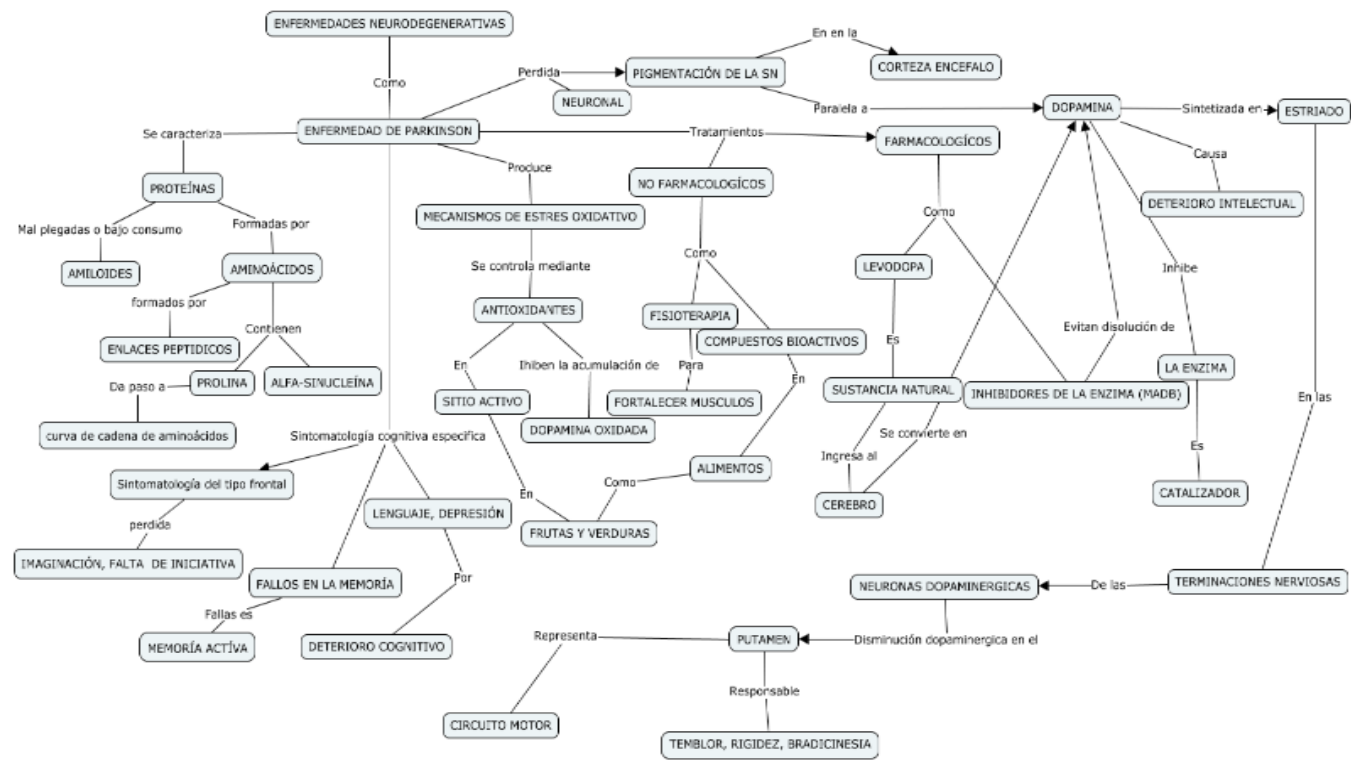

J. Presenta una estructura jerárquica ordenada y clara. Aborda distintos aspectos de la enfermedad, como posibles causas, tratamiento, alimentación y daño neuronal. En su mayoría, las relaciones que presenta son claras y correctas. La relación entre inhibe la enzima y catalizador es incorrecta.

I. Muestra interrelación entre diversos conceptos: la interrelación entre alfa sinucleína, prolina, aminoácidos y curva cadena de aminoácidos se realiza de forma correcta.

N. E. Utiliza frases y palabras conectoras para relacionar cada uno de los conceptos en la mayoría de las veces de forma correcta.

P. Aborda aspectos relacionados con los síntomas cognitivos de forma correcta.

Nota: estudiante [E], jerarquización [J], interrelación [I], explicitación de nexos [N. E.], grado de profundización del contenido $[P]$.

Tabla 12

Resultados de la correlación de Pearson

\begin{tabular}{cccc}
\hline & & Mapa Conceptual & Nivel de argumentación \\
\hline \multirow{2}{*}{ Mapa conceptual } & Correlación de Pearson & 1 &, $413^{*}$ \\
& Sig. (bilateral) & &, 045 \\
\hline Nivel de & N & 24 & 24 \\
argumentación & Sig. (bilateral) &, $413^{*}$ & 1 \\
& $\mathrm{~N}$ &, 045 & 24 \\
\hline
\end{tabular}

Nota: ${ }^{*}$ La correlación es significante al nivel 0.05 (bilateral)

De estos resultados se obtiene que, tanto en el instrumento de entrada como en el final, existe una correlación significativa entre la estructura conceptual y el nivel de argumentación de los estudiantes. No obstante, en el instrumento de entrada se obtuvo una correlación del $99 \%$, mientras que en este instrumento la correlación fue del $95 \%$. Este resultado se explica porque el nivel de argumentación general aumentó a 3 y se evidencia que los 
estudiantes identifican la necesidad de ampliar sus conocimientos para complementar aún más sus argumentos. Así, se evidencia una evolución de la habilidad argumentativa. Sin embargo, a los estudiantes aún se les dificulta realizar operaciones de niveles superiores de argumentación, como construir ordenes de contrademanda o refutar sus propias conclusiones.

En el mapa conceptual fue posible obtener valores superiores a 3, como 3,5 y 4,5, dado que los estudiantes establecieron mejores relaciones entre los conceptos de la química de alimentos y la bioquímica de las END. Tomando como referencia lo establecido por Costamagna (2001) — quien indica que, cuando se da el proceso de aprendizaje, el estudiante reconoce de forma correcta relaciones conceptuales entre conceptos o preposiciones de un conjunto de temas-, se podría decir que los estudiantes comprendieron en gran medida la temática de la química de alimentos y su influencia en las END.

En este sentido, con cada uno de los temas implementados y con los resultados obtenidos, es posible establecer que la unidad didáctica —además de brindar espacios para favorecer el desarrollo de la habilidad argumentativa- permite que conceptos de la bioquímica asociados a la química de alimentos puedan ser enseñados en contextos sociales cercanos a los intereses de los estudiantes, lo cual facilita su comprensión y la aplicabilidad de dichos conceptos al tratar situaciones controvertidas como: prevención y desarrollo de END, alimentos modificados, obtención de fármacos de fuentes naturales y hábitos alimenticios.

\section{Conclusiones}

La unidad didáctica "La alimentación en la prevención y tratamientos de la EP: retos y oportunidades" - gracias a la implementación de actividades como preguntas abiertas, foros de discusión, búsqueda de posibles alternativas en la prevención y en el tratamiento de la EP, entre otras actividades - permitió el fomento de la argumentación. Se evidenció que los estudiantes emplearon los contenidos presentados para reflexionar sobre la situación actual de esta patología, las diferentes implicaciones en la sociedad en general y los retos que conlleva, para construir argumentos en los cuales manifestaban su punto de vista al valerse de componentes científicos, sociales, políticos, económicos y morales para apoyarlos.

Con la implementación del instrumento final, fue posible establecer que los estudiantes en su mayoría lograron alcanzar un nivel dos de argumentación, en el cual respaldaron sus afirmaciones o conclusiones, ya que acudieron a datos de naturaleza social y científica. Así mismo, mediante el cálculo del índice de correlación de Pearson, se estableció que existe una correlación significativa entre la estructura conceptual del estudiante y su nivel de argumentación. Esto podría estar relacionado con el hecho de que pueden expresar su conocimiento tanto de forma representativa como de forma textual.

Las CSC en el aula permiten la construcción y la negociación de argumentos por medio del discurso científico al momento de buscar propuestas o posibles soluciones a situaciones controvertidas, mediante el análisis de la información, la búsqueda de evidencia, la contrademanda de argumentos y la justificación del conocimiento.

Ya que a nivel mundial no existen referentes en los cuales se aborde la alimentación en las END como CSC para desarrollar la habilidad argumentativa, este trabajo es pionero en este contexto. Se cumplió el objetivo principal de favorecer la habilidad argumentativa, pero 
además hubo una contribución conceptual al entendimiento de temáticas como la química de alimentos en las END.

\section{Referencias}

Costamagna, A. (2001). Mapas conceptuales como expresión de procesos de interrelación para evaluar la evolución del conocimiento de alumnos universitarios. Enseñanza de las Ciencias: Revista de Investigación y Experiencias Didácticas, 19(2), 309-318. https://www. raco.cat/index.php/Ensenanza/article/view/21749

Enfermedad ParkinsonCSC. (2019). Enfermedad de Parkinson [Video]. YouTube. https:// www.youtube.com/watch?v=kTqnQzFO-Bw\&t=71s

Fernández, R., Gasca, C., Sánchez, Á., \& Obeso, J. (2016). Actualización en la enfermedad de Parkinson. Revista Médica Clínica Las Condes, 27(3), 363-379. https://doi.org/10.1016/j. rmclc.2016.06.010

Garcés, M. (2016). Estudio sobre las enfermedades neurodegenerativas en España y su impacto económico y social. Gobierno de España.

HablemosClaroTV. (2017). Alimentos funcionales [Video]. Youtube. https://www.youtube. com/watch?v=wnLBEFJ1Av0

Hernández, R., Fernández, C., \& Baptista, P. (2017). Metodología de la investigación. McGraw Hill.

Jiménez-Aleixandre, M., \& Erduran, S. (2007). Argumentation in Science Education: An Overview. En M. Jiménez-Aleixandre, \& S. Erduran (Eds.), Argumentation in Science Education (pp. 3-27). Science \& Technology Education Library.

Martín-Gámez, C., \& Erduran, S. (2018). Understanding argumentation about socioscientific issues on energy: a quantitative study with primary pre-service teachers in Spain. Research in Science \& Technological Education, 34(4), 463-483. https://doi.org/10 .1080/02635143.2018.1427568

Mesa, L. (2010). Propuesta estrategia educativa para familiares y/o cuidadores de pacientes con enfermedad de parkinson ejecutada por profesionales del area de nutrición y dietética [Trabajo de grado, Pontificia Universidad Javeriana]. Repositorio Institucional Pontificia Universidad Javeriana. https://repository.javeriana.edu.co/bitstream/ handle/10554/8792/tesis737.pdf

Osborne, J., Erduran, S., \& Simon, S. (2004). Enhancing the quality of argument in school science. Journal of Research in Science Teaching, 41(10), 994-1020. https://doi.org/ 10.1002/tea.20035

Posada, J. (2015). La argumentación y su rol en el aprendizaje de la ciencia. Revista Tesis Psicológica, 10(1), 146-160.

Reguant, M., Vilà, R., \& Torrado, M. (2018). La relación entre dos variables según la escala de medición con SPSS. Revista d'Innovació i Recerca en Educació, 11(2), 45-60.

Sadler, T. (2009). Situated learning in science education: socio-scientific issues as contexts for practice. Studies in Science Education, 45(1), 1-42. https://doi. org/10.1080/03057260802681839 